\title{
FOURIER TRANSFORMS OF UNBOUNDED MEASURES ${ }^{1}$
}

\author{
BY LOREN ARGABRIGHT AND JESÚS GIL DE LAMADRID
}

Communicated by Ray A. Kunze, November 12, 1970

Let $G$ be a locally compact abelian group. For a (generally unbounded) measure $\mu$ on $G$ we shall say that $\mu$ is transformable if there is a measure $\hat{\mu}$ on the character group $\Gamma$ of $G$ such that, for every $f \in K(G)$, the space of continuous functions with compact support on $G, \hat{f} \in L_{2}(\hat{\mu})$ and

$$
\int_{G} f^{*} * f(x) d \mu(x)=\int_{\Gamma}\left|\hat{f}\left(\gamma^{-1}\right)\right|^{2} d \hat{\mu}(\gamma) .
$$

The resulting "Fourier transformation" $\mu \rightarrow \hat{\mu}$ contains the classical theory and leads to generalizations of a variety of classical results, including the Plancherel theorem and the Poisson summation formula. The present work can also be regarded as a sort of theory of tempered distributions on general locally compact abelian groups. It is true that Bruhat [11] introduced a direct generalization to this setting of the theory of Schwartz [10], but, to the best of our knowledge, a detailed study of the Fourier transform has not been carried out. In a forthcoming exposition we shall describe the precise relation between the present study and the work of Bruhat.

In the present announcement of results, we shall, for the most part, restrict ourselves to the study of the Fourier transform on the linear span $\mathfrak{T}(G)$ of all positive definite measures on $G$. Such a measure $\mu$ is defined by the property: $\mu\left(f * f^{*}\right) \geqq 0$, for $f \in K(G)$. The transformability of the elements of $\mathfrak{m}(G)$ follows from a general Plancherel theorem of Godement [6, p. 144-07]. ${ }^{2}$ If $\mu$ is the Dirac measure at the identity, Godement's theorem reduces to the classical Plancherel theorem for $G$. In a similar spirit, a general Poisson summation formula can be established for positive definite $\mu$, which reduces to the classical formula in the case that $\mu$ is Haar measure on a closed subgroup of $G$. This is the content of Theorem 6 below.

AMS 1970 subject classifications. Primary 43A05, 43A25, 43A32; Secondary 43A35, 43A65.

Key words and phrases. Translation bounded measure, transformable measure, positive definite measure, Fourier transformation, Poisson summation formula.

1 Partial support of this work by NSF (GP 7678, GP 12361) is gratefully acknowledged.

${ }^{2}$ We are indebted to the referee for bringing this reference to our attention. This made it possible to shorten a previous version of our announcement. 
1. Transforms of measures in $\operatorname{Tr}(G)$. We shall pick and fix a Haar measure $d x$ on $G$, and, without further ado, identify a locally integrable function $f$ on $G$ with the measure $f(x) d x$. If $f$ is continuous and $\mu$ is a (not necessarily bounded) measure on $G, f \mu$ shall, as usual, denote the measure $f(x) d \mu(x)$. The transformability of positive definite measures, and the uniqueness part of the theorem of Godement, cited above, yields a mapping $\mu \rightarrow \hat{\mu}$ of positive definite measures on $G$ to positive measures on $\Gamma$, which can be extended by linearity to the entire linear span $\operatorname{IR}(G)$ of the set of positive definite measures. The precise details are given by the following theorem, whose proof is like the uniqueness part of the theorem of Godement.

ThEOREM 1. Every $\mu \in \mathfrak{T}(G)$ is transformable and the measures $\mu$ and $\hat{\mu}$, satisfying (1), with $\mu \in \mathscr{T}(G)$, determine each other uniquely. Thus, the mapping $\mu \rightarrow \hat{\mu}$ is linear and one-to-one on $\mathfrak{T l}(G)$.

If $\mu \in \mathfrak{M}(G)$ we shall henceforth refer to $\hat{\mu}$ as the Fourier transform of $\mu$. The following theorem justifies our claim that the Fourier transform in this general setting includes the various classical notions that are commonly considered. As in [8], we use $B(G)$ to denote the algebra of Fourier-Stieltjes transforms of bounded measures on $\Gamma$. Thus, if $g \in B(G)$, there is a unique bounded measure $\theta_{0}$ on $\Gamma$ such that $g(x)=\int_{\Gamma} \gamma(x) d \theta_{o}(\gamma)$; we shall refer to $\theta_{g}$ as the Bochner measure of $g$.

THEOREM 2. The space $\operatorname{Tr}(G)$ contains $L_{p}(G)(1 \leqq p \leqq 2), M(G)$ and $B(G)$. Furthermore, the transform $\mu \rightarrow \hat{\mu}$ extends the classical Fourier transform on $L_{p}(G)(1 \leqq p \leqq 2)$, and the Fourier-Stieltjes transform on $M(G)$, and assigns to $g \in B(G)$ its Bochner measure.

We now turn to the problem of finding an inversion formula for the transform $\mu \rightarrow \hat{\mu}$. In this regard, a natural question is whether the transform $\hat{\mu}$ of a measure $\mu$ in $\operatorname{Tr}(G)$ belongs to $\operatorname{Tl}(\Gamma)$. Unfortunately, this is not true in general. In view of this, it is natural to introduce a new space of measures, namely $\mathfrak{g}(G)=\{\mu \in \mathfrak{M}(G): \hat{\mu} \in \mathfrak{M}(\Gamma)\}$. Clearly $\mathfrak{g}(G)$ is a linear subspace of $\mathfrak{T l}(G)$. Furthermore, one can show that, like $\mathscr{T}(G), \mathscr{g}(G)$ contains the spaces $L_{p}(G)(1 \leqq p \leqq 2), M(G)$, and $B(G)$, as well as the Haar measures of closed subgroups of $G$.

For $f \in K(G)$, we define $f^{\prime}$ by the formula $f^{\prime}(x)=f\left(x^{-1}\right)$. For a measure $\mu, \mu^{\prime}$ is defined by transposition, that is $\mu^{\prime}(f)=\mu\left(f^{\prime}\right)$. If $\mu$ is a measure in $\mathfrak{N}(G)$ (or $\mathfrak{T}(\Gamma)$ ), we define $\check{\mu}=\hat{\mu}^{\prime}$, and we call $\check{\mu}$ the inverse Fourier transform of $\mu$. We see immediately that, for $\mu \in \mathscr{T}(G)$, $\mu^{\prime} \in \mathscr{M}(G)$ and $\left(\mu^{\prime}\right)^{\wedge}=(\hat{\mu})^{\prime}$. Thus $\mathscr{M}(G)$ and $\mathscr{g}(G)$ are invariant under reflection. 
THEOREM 3. The Fourier transform establishes an isomorphism of the space $\mathscr{J}(G)$ onto the space $\mathscr{S}(\Gamma)$ whose inverse is the inverse Fourier transform, that is, for every $\mu \in \mathcal{I}(G), \mu=\hat{\mu}^{2}$.

2. Stability properties of $\operatorname{Tr}(G)$. The considerations of this section involve convolutions of measures which, in general, are unbounded. For the definition of convolution in the generality needed here we refer to Bourbaki [1]. In discussing the convolution of general measures, one of the main concerns, of course, is with the question of when the convolution is defined. Classically it is well known that $\mu * \nu$ exists if one of the measures has compact support, or if both are bounded. In addition, certain unbounded measures (for example $d x$ ) can be convolved with any bounded measure. To some extent, the measures under consideration in this paper are inclined to have this curious property. It turns out, for example, that the transform $\hat{\mu}$ of any measure $\mu \in \mathscr{I}(G)$ can be convolved with any bounded measure on $\Gamma$; however, the corresponding statement for $\mu$ is not true in general.

Theorem 4. (a) If $g \in B(G)$ and $\mu \in \mathscr{T l}(G)$ then $g \mu \in \mathscr{T l}(G)$ and $(g \mu)^{\wedge}=\theta_{g} * \hat{\mu}$ (in particular, this convolution exists).

(b) If $\mu \in \mathfrak{T}(G)$, and if $\nu$ is a measure having compact support, then $\nu * \mu \in \mathfrak{T}(G)$ and $(\nu * \mu)^{\wedge}=\hat{\nu} \hat{\mu}$ (keep in mind here that $\hat{\nu}$ is a function).

We note that it follows from (a) that $\mathfrak{T}(G)$ is stable under multiplication by characters, and that multiplication of $\mu$ by a character $\gamma$ corresponds to translation of $\hat{\mu}$ by $\gamma$. We also see, from (b), that $\mathscr{T M}(G)$ is translation invariant (convolve $\mu$ with a point mass) and that translation of $\mu$ corresponds to multiplication of $\hat{\mu}$ by the appropriate character. For the sake of comparison, it is also interesting to note that Theorem 4 tells us that $\widehat{\mathscr{N}}(G)$ is stable under convolution by bounded measures and under multiplication by Fourier-Stieltjes transforms of measures with compact support. Finally, we point out that the space $\mathscr{g}(G)$ introduced in $\$ 1$ has all the stability properties in question here. This follows from Theorems 3 and 4, and from the fact that the convolution of two positive definite measures (if it exists) is also positive definite.

THEOREM 5. The space $\mathfrak{J}(G)$ is stable under convolution by arbitrary bounded measures and under multiplication by arbitrary FourierStieltjes transforms.

3. The general Poisson summation formula. Let $H$ be a closed subgroup of $G$ with Haar measure $d x_{H}$. One verifies easily that $d x_{H}$ (regarded as a measure on $G$ ) is positive definite, hence transformable. 
One shows (for instance, by using the classical Poisson summation formula) that its transform, denoted by $d \gamma_{H^{0}}$, is a Haar measure on the annihilator $H^{0}$ of $H$ in $\Gamma$. Setting $f=g * g^{*}$, for $g \in K(G)$, formula (1) yields, using the invariance of Haar measure with respect to reflection,

$$
\int_{H} f(x) d x_{H}=\int_{H^{0}} \hat{f}(\gamma) d \gamma_{H^{0}} .
$$

Thus formula (1) appears as the exact analogue, for arbitrary transformable measures, of the classical Poisson formula, and of the usual Fourier inversion formula, in the case $H=\{e\}$. The usual question that is asked about formula (2) concerns the extent to which it remains valid for a larger class of functions. We are able to prove certain results of this type, for arbitrary positive definite $\mu$, which, when applied to the case $d \mu(x)=d x_{H}$, includes all the classical results that are known to us (see, for example, [2], [3], [7], and [9]).

Before stating our theorem, we need to say a few words about convolution of functions and measures. In the first place, if $f$ is a locally integrable function and $\mu$ is a measure, then the convolution $f * \mu$ is to be interpreted in the sense of measures, i.e. as the convolution of the measures $f(x) d x$ and $\mu$. This convolution, when it exists, is given by a locally integrable function and, under suitable additional assumptions (see [1] or [4]), one has the formula

$$
f * \mu(x)=\int_{G} f\left(y^{-1} x\right) d \mu(y)
$$

locally almost everywhere.

THEOREM 6. Let $\mu$ be a positive definite measure on $G$ and let $f$ be a Borel measurable function belonging to $L_{1}(G)$. Suppose $f^{\prime} * \mu$ exists and, further, that the integral $\int_{G} f\left(y x^{-1}\right) d \mu(y)$ exists for $x$ in a neighborhood of the identity and is a continuous function of $x$ at $x=e$. Then:

(a) if $\hat{f}^{\prime} \in L_{1}(\hat{\mu})$ we have

$$
\int_{G} f(y) d \mu(y)=\int_{\Gamma} \hat{f}\left(\gamma^{-1}\right) d \hat{\mu}(\gamma),
$$

(b) if $f$ is continuous and positive definite then $\hat{f}^{\prime} \in L_{1}(\hat{\mu})$ and (4) holds.

We remark, finally, that in the case $d \mu=d x_{H}$ the condition that $f^{\prime} * \mu$ exists is automatically satisfied (because $d x_{H}$ belongs to $\widehat{\mathscr{N}}(\Gamma)$ ). However, the assumption that the convolution be continuous at $e$ still seems to be necessary. 


\section{REFERENCES}

1. N. Bourbaki, Livre VI: Intégration. Chaps. 1, 8, Actualités Sci. Indust., nos. 1175, 1306, Hermann, Paris, 1965, 1963. MR 31 \#3539; MR 36 \#2763.

2. —, Théories spectrales, Chaps. 1, 2, Actualités Sci. Indust., no. 1332, Hermann, Paris, 1967. MR $35 \# 4725$.

3. S. Bochner, Vorlesungen über Fouriersche Integrale, reprint, Chelsea, New York, 1948; English transl., Ann. of Math. Studies, no. 42, Princeton Univ. Press, Princeton, N. J., 1959.

4. E. Hewitt and K. Ross, Abstract harmonic analysis. Vol. I: Structure of topological groups. Integration theory, group representation, Die Grundlehren der math. Wissenschaften, Band 115, Academic Press, New York; Springer-Verlag, Berlin, 1963. MR 28, \#158.

5. J. Glimm, Lectures on harmonic analysis, Courant Institute Notes, New York University, New York, 1965.

6. R. Godement, Introduction aux travaux de A. Selberg, Séminaire Bourbaki, 1956/57, Exposé 144, Sécrétariat Mathématique, Paris, 1959. MR 28 \#1090.

7. L. Loomis, An introduction to abstract harmonic analysis, Van Nostrand, Princeton, N. J., 1953. MR 14, 883.

8. W. Rudin, Fourier analysis on groups, Interscience Tracts in Pure and Appl. Math., no. 12, Interscience, New York, 1962. MR 27 \#2808.

9. H. Reiter, Classical harmonic analysis and locally compact groups, Oxford Math. Monographs, Clarendon Press, Oxford, 1968.

10. L. Schwartz, Thêorie des distributions, new ed., Hermann, Paris, 1966. MR 35 \#730.

11. F. Bruhat, Distributions sur un groupe localement compact et applications a l'étude des représentations des groupes p-adiques, Bull. Soc. Math. France 89 (1961), 43-75. MR 25 \#4354.

UNIVERSity OF Nebraska, LiNCOLN, Nebraska 68508

University of Minnesota, Minneapolis, Minnesota 55455 